\title{
Impact of Socioeconomic Status on Adult Patients with Asthma: A Population-Based Cohort Study from UK Primary Care
}

\author{
John Busby, ' David Price, (iD) ${ }^{2-4}$ \\ Riyad Al-Lehebi, ${ }^{5}$ Sinthia \\ Bosnic-Anticevich, (iD) ${ }^{6-8}$ \\ Job FM van Boven, (D) 9 \\ Benjamin Emmanuel, ${ }^{10}$ \\ J Mark FitzGerald, '1 Mina Gaga, ${ }^{12}$ \\ Susanne Hansen, ${ }^{13,14}$ Mark Hew, ${ }^{15,16}$ \\ Takashi Iwanaga, (iD) ${ }^{17}$ \\ Désirée Larenas Linnemann, (iD ${ }^{18}$ \\ Bassam Mahboub, ${ }^{19,20}$ Patrick Mitchell, ${ }^{21}$ \\ Daniela Morrone, ${ }^{22}$ Jonathan Pham, ${ }^{15,23}$ \\ Celeste Porsbjerg, ${ }^{14}$ Nicolas Roche, (ID) 24,25 \\ Eileen Wang, ${ }^{26,27}$ Neva Eleangovan, ${ }^{2,3}$ \\ Liam G Heaney ${ }^{28}$
}

'Centre for Public Health, Queen's University Belfast, Belfast, Northern Ireland; ${ }^{2}$ Optimum Patient Care, Cambridge, UK; ${ }^{3}$ Observational and Pragmatic Research Institute, Singapore, Singapore; ${ }^{4}$ Centre of Academic Primary Care, Division of Applied Health Sciences, University of Aberdeen, Aberdeen, UK; ${ }^{5}$ Department of Pulmonology, King Fahad Medical City, Riyadh, Saudi Arabia; ${ }^{6}$ Sydney Pharmacy School, Faculty of Medicine and Health, The University of Sydney, Sydney, Australia; ${ }^{7}$ Woolcock Institute of Medical Research, The University of Sydney, Sydney, Australia; ${ }^{8}$ Central Sydney Local Area Health District, Sydney, Australia; ${ }^{9}$ Department of Clinical Pharmacy \& Pharmacology, Groningen Research Institute for Asthma and COPD (GRIAC), University Medical Center

Groningen, University of Groningen,

Groningen, the Netherlands;

${ }^{10}$ BioPharmaceuticals Medical, AstraZeneca, Gaithersburg, MD, USA; ' 'Department of Medicine, The University of British Columbia, Vancouver, Canada; ${ }^{12} 7$ th Respiratory Medicine Department and Asthma Centre, Athens Chest Hospital, Athens, Greece; ${ }^{13}$ Center for Clinical Research and Prevention, Bispebjerg and Frederiksberg Hospital, Copenhagen, Denmark;

${ }^{14}$ Respiratory Research Unit, Bispebjerg

University Hospital, Copenhagen, Denmark;

${ }^{15}$ Allergy, Asthma \& Clinical Immunology

Service, Alfred Health, Melbourne, Australia;

${ }^{16}$ Public Health and Preventive Medicine, Monash University, Melbourne, Australia;

${ }^{17}$ Center for General Medical Education and

Clinical Training, Kindai University Hospital,

Osakasayama, Japan; ${ }^{18}$ Directora Centro de

Excelencia en Asma y Alergia, Hospital Médica

Sur, Ciudad de México, Mexico; ${ }^{19}$ College of
Introduction: Asthma morbidity and health-care utilization are known to exhibit a steep socioeconomic gradient. Further investigation into the modulators of this effect is required to identify potentially modifiable factors.

Methods: We identified a cohort of patients with asthma from the Optimum Patient Care Research Database (OPCRD). We compared demographics, clinical variables, and health-care utilization by quintile of the UK 2011 Indices of Multiple Deprivation based on the location of the patients' general practice. Multivariable analyses were conducted using generalized linear models adjusting for year, age, and sex. We conducted subgroup analyses and interaction tests to investigate the impact of deprivation by age, sex, ethnicity, and treatment step.

Results: Our analysis included 127,040 patients with asthma. Patients from the most deprived socio-economic status (SES) quintile were more likely to report uncontrolled disease (OR: 1.54, 95\% CI: 1.16, 2.05) and to have an exacerbation during follow-up (OR: 1.27, 95\% CI: 1.13, 1.42) than the least deprived quintile. They had higher blood eosinophils (ratio: 1.03; 95\% CI: 1.00, 1.06 ) and decreased peak flow (ratio: $0.95,95 \%$ CI: 0.94, 0.97) when compared to those in the least deprived quintile. The effect of deprivation on asthma control was greater among those aged over 75 years $(\mathrm{OR}=1.81,95 \% \mathrm{CI}: 1.20,2.73)$ compared to those aged less than 35 years (OR: $1.22,95 \%$ CI: $\left.0.85,1.74 ; p_{\text {interaction }}=0.019\right)$. Similarly, socioeconomic disparities in exacerbations were larger among those from ethnic minority groups (OR: 1.94, 95\% CI: 1.40, 2.68) than white patients (OR: $1.24,95 \%$ CI: $1.10,1.39$; $p_{\text {interaction }}=0.012$ ).

Conclusion: We found worse disease control and increased exacerbation rates among patients with asthma from more deprived areas. There was evidence that the magnitude of socioeconomic disparities was elevated among older patients and those from ethnic minority groups. The drivers of these differences require further exploration.

Keywords: asthma, socioeconomic status, disparities

\section{Introduction}

Socioeconomic status (SES) is a multi-faced construct comprising elements including education, housing, and living environment. Within England, there is an almost two-decade gap in healthy life expectancy between those living in the least and most deprived areas, and socioeconomic inequalities continue to widen. ${ }^{1}$ Asthma is the second most common chronic respiratory disease worldwide, affecting around 300 million patients and costing in excess of $\$ 80$ billion annually in the United States (US) alone. ${ }^{2,3}$ Although the disease is prevalent across society, it is known to disproportionally affect deprived populations, ${ }^{4,5}$ and exploring the interaction between socioeconomic factors and asthma outcomes has recently been identified 
Medicine, University of Sharjah, Sharjah, United Arab Emirates; ${ }^{20}$ Rashid Hospital, Dubai Health Authority, Dubai, United Arab Emirates; ${ }^{21}$ Cumming School of Medicine, University of Calgary, Calgary, Alberta, Canada;

${ }^{22}$ Personalized Medicine, Asthma and Allergy, Humanitas Clinical and Research Center, IRCCS, Rozzano, Italy; ${ }^{23}$ Allergy and Lung Health Unit, Centre for Epidemiology and Biostatistics, Melbourne School of Population \& Global Health, The University of Melbourne, Melbourne, Australia; ${ }^{24}$ Respiratory Medicine, Cochin Hospital, APHP.Centre, Paris, France;

${ }^{25}$ University of Paris, Cochin Institute (UMR I0I6), Paris, France; ${ }^{26}$ Division of Allergy \& Clinical Immunology, Department of Medicine, National Jewish Health, Denver, CO, USA; ${ }^{27}$ Division of Allergy \& Clinical Immunology, Department of Internal Medicine, University of Colorado School of Medicine, Aurora, CO, USA; ${ }^{28}$ Wellcome-Wolfson Centre for Experimental Medicine, Queen's University Belfast, Belfast, Northern Ireland

Correspondence: David Price Centre of Academic Primary Care, Division of Applied Health Sciences, University of Aberdeen, Polwarth Building, Foresterhill, Aberdeen, AB25 2ZD, UK

Tel +65 69623627

Email dprice@opri.sg as a key research priority. ${ }^{6}$ Several potential mechanisms for worse asthma outcomes among deprived populations have been suggested including higher smoking rates, poorer housing conditions, occupational exposures, less effective self-management, and an inability to afford medications. ${ }^{7,8}$

Much of the previous literature investigating the social determinants on asthma morbidity and mortality have focused on the US, perhaps due to the stark divide in health-care accessibility driven by variable health-care insurance coverage. ${ }^{9}$ Although these studies are heterogeneous in their populations and methods, they have consistently reported higher rates of morbidity and unscheduled care use among more deprived populations. ${ }^{10-12}$ Studies from outside the US are less common, however poorer asthma outcomes have been reported among deprived populations across several countries, including those with universal health-care coverage such as Sweden and Canada. ${ }^{13,14}$ Studies from the UK are sparse, with most relevant evidence being drawn from research conducted more than ten years ago. ${ }^{15-17}$ One recent ecological study did report substantially higher hospital admissions in more deprived areas while also noting a strong moderating effect of age on mortality. ${ }^{18}$ This finding raises the question of whether other facets of asthma care, including symptom control and exacerbation frequency, are similarly modulated by patient demographics and clinical factors.

Here, we provide updated evidence on the magnitude of socioeconomic disparities within the United Kingdom (UK). We investigate factors that may modulate the SES effect and explore potential mechanisms driving disparities, which is necessary to design effective interventions.

\section{Materials and Methods}

\section{Study Population}

Data were extracted from the Optimum Patient Care Research Database (OPCRD; https://opcrd.co.uk/), which is a nationally representative pseudonymized dataset comprising information on patient demographics, clinical diagnoses, medication prescriptions, and referrals coded through the Read and SNOMED classification systems. At data extraction, OPCRD contained data for approximately 9.7 million patients registered at 700 general practices within the UK ( $8 \%$ of the UK population). ${ }^{19}$ Read codes are a hierarchical clinical terminology used within UK general practice for over 20 years that have recently been replaced by SNOMED codes, which serve broadly the same purpose. ${ }^{20}$ Patient data became eligible for analysis at the latest date of: 1) 1st April 2008 [as electronic prescription recording was less common before this date], 2) when the patient was diagnosed with asthma [using a validated list of read $\operatorname{codes}^{21}$ ], 3) when the patient turned 18 years old, 4) when three years of data were available for the patient [to allow time for potential confounder ascertainment]. Patient data were followed-up until the earliest date of: 1) when the patient left the practice, 2) when data were last collected from the practice, 3 ) when the patient's asthma was recorded as resolved (Read Code: 21262).

To prevent time-window bias, ${ }^{22}$ we used a standard one-year window to assess outcomes for all patients. Those with less than one year of eligible follow-up time were excluded from the study. We randomly chose a one-year ascertainment period for patients with more than one year's eligible follow-up time. To increase the comparability of our cohort, those with an alternative respiratory diagnosis (chronic obstructive pulmonary disease, cystic fibrosis, idiopathic pulmonary fibrosis,pulmonary sarcoidosis, or interstitial pneumonia) 
in the three years prior to inclusion, and those who were not receiving active treatment for their asthma in the last year (at least one prescription of short-acting $\beta$-agonist [SABA], inhaled corticosteroids [ICS], long-acting $\beta$-agonist [LABA], leukotriene receptor antagonists [LTRA], oral corticosteroids [OCS]), were excluded from the analysis.

\section{Exposures}

Socioeconomic status was determined using the UK 2011 Indices of Multiple Deprivation (IMD). The summary IMD score calculates the relative deprivation of small areas by taking a weighted average across seven domains (income, employment, health, education, housing, crime, and living environment). ${ }^{23}$ Each patient's IMD score was based on the location of their general practice; we aimed to use this as a proxy measure for individual SES. Within the UK, almost all patients are registered with a general practice that provides primary care, including disease diagnosis, monitoring and prevention. We grouped patients into five deprivation quintiles ranging from least deprived (quintile 5) to most deprived (quintile 1).

\section{Outcomes}

We investigated differences in asthma presentation (blood eosinophils, peak flow), processes (treatment adherence, asthma reviews, respiratory referrals) and health-care outcomes (asthma control, exacerbations) using data measured during the yearlong ascertainment period described previously. Peak flow was calculated using raw measurements and the formula specified by Knudson et al. ${ }^{24}$ This adjusted for age, sex and height, although did not account for ethnicity, and we are unaware of any ethnicity-adjusted peak flow reference values that can be appropriately applied to the UK population. We used a percent predicted peak flow value recorded directly in the medical records when no raw peak flow measure was available, or when the patient's height was unavailable. Medication adherence was assessed using the fixed medication possession ratio (MPR) of ICS during the ascertainment period. Good adherence was defined as an MPR of greater than or equal to $70 \%$. Asthma reviews and respiratory referrals were identified using Read code lists. ${ }^{25}$ Asthma control was measured using the Royal College of Physicians 3 questions. ${ }^{26}$ Asthma exacerbations were identified using either a Read code indicating an "Asthma Exacerbation" or "Asthma Attack", prescription of acute oral corticosteroids, or a lower respiratory infection requiring antibiotics. Full details of all outcomes are provided in Online Supplement 1.

\section{Covariates}

Patient sex and year of birth were supplied directly from the general practice for all patients. Other patient information including ethnicity (categorized using UK census definitions: White, Mixed, Asian, Black, Other), body mass index (BMI), and smoking status were derived using Read codes recorded prior to the start of follow-up. We also used Read codes to identify comorbidities (Allergic Rhinitis, Cancer, Cataract, Cerebrovascular Disease, Congestive Heart Disease, Depression/Anxiety, Diabetes, Eczema, Glaucoma, Hypertension, Insomnia, Liver Disease, Myocardial Infarction, Nasal Polyps, Oral Candidiasis, Osteoporosis, Renal Disease, Rheumatological Disease) using a list based on the Charlson comorbidity index, ${ }^{27}$ depression $^{28}$ and those related to systemic corticosteroid exposure. ${ }^{29}$ Asthma medications were identified using Read/SNOMED hierarchies, and patients were categorized according to Global Initiative for Asthma (GINA) 2018 treatment step ${ }^{30}$ (Online Supplement 2). ICS dose was converted to a beclomethasone dipropionate (BDP) equivalent. ${ }^{31}$ Step five was defined as more than 6 prescriptions of OCS in a year, spanning across at least two quarters. ${ }^{32}$ In general, covariates were ascertained in the year prior to the outcome ascertainment period. Full details are provided in Online Supplement 1.

\section{Statistical Analysis}

Demographic and clinical outcomes were analyzed descriptively and comparisons across IMD quintiles were undertaken. Univariate analyses were conducted using t-tests, chi-square tests and Mann-Whitney $U$-tests where appropriate. Multivariable analyses were conducted adjusting for year, age (5-year categories) and sex to address potential cofounding by these factors. We chose this limited set of adjustment variables to prevent any overadjustment bias, whereby adjustment is made for variables that lie on the causal path between SES and outcomes, to ensure that we were capturing the full magnitude of any disparities. $^{33}$ Binary outcome variables were modelled using logistic regression, and continuous data was modelled using gamma generalized linear models with a log link function. Consequently, all multivariable results are shown as odds ratios or ratios. The clustering of patients 
within GP practices was accounted for using cluster robust standard errors.

\section{Subgroup and Sensitivity Analyses}

We investigated the extent to which demographic factors (age, sex, and ethnicity) or treatment step (used as a marker of asthma severity ${ }^{34}$ ) modulated the effect of deprivation on peak flow, asthma disease control, and exacerbations. We repeated our regression analysis stratified for each subgroup (eg, male vs female) and plotted odds ratios comparing IMD quintiles. The ethnicity analysis combined all ethnic minority groups (EMGs; Asian, Black, mixed, or other) vs White patients to maximize statistical power. We performed formal hypothesis tests using interaction tests investigating whether the difference between the least and most deprived quintiles varied across subgroups. We did not conduct subgroup analysis for other outcomes that were related to intermediate factors (blood eosinophils, treatment adherence) or process measures (asthma review or respiratory referral).

Our primary multivariable analysis was based on complete cases (ie, only patients with complete data for all variables in the model) however we used multiple imputation with chained equations, which assumes that the data was missing at random, to assess the impact of missing data. ${ }^{24}$ Ten imputation datasets were created, and imputation models including year, age, sex and ethnicity. To investigate if the effect of socioeconomic status was mediated by lifestyle factors, we reran our multivariable regression models investigating socioeconomic disparities in blood eosinophils, peak flow, treatment adherence, asthma reviews, respiratory referrals, asthma control and exacerbations additionally adjusting for smoking status and BMI category $(<18.5$, 18.5-25.0, $25.1-30.0,>30 \mathrm{~kg} / \mathrm{m}^{2}$ ). UK guidelines state that patients with frequent use of oral corticosteroid should be referred for specialist review. ${ }^{35}$ Therefore, we additionally investigated the impact of SES on referral rates among patients who had at least two exacerbations in the previous year.

\section{Results}

\section{Cohort Demographics and Univariate Comparison by IMD Quintiles}

Our analyses included 127,040 patients with 28,215 and 16,534 from the least and most deprived quintiles, respectively. Mean patient age was 51.2 years, and a female predominance $(59.1 \%)$ was evident across all SES groups (Table 1). There was a strong relationship between ethnicity and SES, with $97.5 \%$ of patients in the least deprived quintile being White compared to $88.3 \%$ within the most deprived quintile $(\mathrm{p}<0.001)$. Patients from the least deprived quintile were more likely to be never smokers (59.2\% vs 50.8\%, p < 0.001); however, they consumed more alcohol (4 vs 2 weekly units, p < 0.001).

In general, the patients in the lowest IMD quintile had higher rates of comorbidities including depression/anxiety $(12.2 \%$ vs $9.3 \%, \mathrm{p}<0.001)$ and diabetes $(8.4 \%$ vs $5.7 \%$, p $<0.001)$, although rates of cancer were lower $(8.3 \%$ vs $12.1 \%, \mathrm{p}<0.001)$ when compared to the least deprived quintile. Medication patterns were similar across IMD quintiles (Online Supplement 3). Patients from the most deprived areas had lower peak flow $(84.9 \%$ vs $89.0 \%$ percent predicted, $\mathrm{p}<0.001)$ and were more likely to report uncontrolled disease $(44.7 \%$ vs $34.5 \%, \mathrm{p}<0.001)$, and a larger proportion experienced an exacerbation during follow-up (24.2\% vs $20.3 \%, \mathrm{p}<0.001)$. Most patients had data available on their age (100\%), smoking status (96.4\%), and medication records $(81.5 \%)$; however, data were less commonly available for several other measures, including blood eosinophils (36.0\%) and disease control (31.5\%; Online Supplement 4).

\section{Multivariate Comparison by IMD Quintiles}

In multivariable analyses, there were substantial differences by IMD quintile for several measures that generally followed a dose-response pattern (Figure 1). Patients in the most deprived quintile had higher blood eosinophils (ratio: 1.03; $95 \%$ CI: $1.00,1.06$ ) and worse percent predicted peak flow (ratio: $0.95,95 \%$ CI: $0.94,0.97$ ). The proportion achieving good medication adherence to ICS was higher among the most deprived group (odds-ratio [OR]: 1.43; 95\% CI: 1.17, 1.76), although there was no evidence that asthma reviews (OR: 0.85 ; $95 \%$ CI: $0.55,1.34$ ) or respiratory referrals (OR: 1.02 ; $95 \%$ CI: $0.55,1.90)$ varied by SES. Patients from the most deprived quintile were substantially more likely to report uncontrolled disease (OR: 1.54, 95\% CI: 1.16, 2.05) and to have an exacerbation during the year-long follow-up period (OR: $1.27,95 \%$ CI: 1.13, 1.42) when compared to the least deprived group.

\section{Modulation of SES Effect by Age and Ethnicity}

There was evidence that the magnitude of socioeconomic disparities varied within patient subgroups. Although increased deprivation was associated with uncontrolled disease across all age groups (Figure 2), it was more 
Table I Comparison of Demographic and Clinical Information by Indices of Multiple Deprivation Quintile

\begin{tabular}{|c|c|c|c|c|c|c|}
\hline & $\begin{array}{l}5 \text { (Least } \\
\text { Deprived) }\end{array}$ & 4 & 3 & 2 & $\begin{array}{c}\text { I (Most } \\
\text { Deprived) }\end{array}$ & $\begin{array}{c}\text { P- } \\
\text { value }\end{array}$ \\
\hline Number Patients & 28,215 & 26,900 & 24,332 & 31,059 & 16,534 & \\
\hline Age (years) & $51.8(16.7)$ & $51.1(17.0)$ & $52.1(17.1)$ & $50.8(17.0)$ & $50.0(16.9)$ & $<0.001$ \\
\hline$<35$ & 4911 (17.4\%) & 5234 (19.5\%) & 4453 (I8.3\%) & 6330 (20.4\%) & 3708 (22.4\%) & \\
\hline $35-54$ & II,553 (40.9\%) & $10,795(40.1 \%)$ & 9369 (38.5\%) & 12,350 (39.8\%) & 6487 (39.2\%) & \\
\hline $55-74$ & 8981 (31.8\%) & $8286(30.8 \%)$ & 7994 (32.9\%) & 9556 (30.8\%) & 4911 (29.7\%) & \\
\hline $75+$ & 2770 (9.8\%) & 2585 (9.6\%) & $2516(10.3 \%)$ & $2823(9.1 \%)$ & I 428 (8.6\%) & \\
\hline Sex & & & & & & $<0.001$ \\
\hline Female & $16,362(58.0 \%)$ & $15,780(58.7 \%)$ & 14,388 (59.1\%) & 18,617 (59.9\%) & $996 \mid$ (60.2\%) & \\
\hline Male & II,853 (42.0\%) & $1 \mathrm{I}, \mathrm{I} 20(4 \mathrm{I} .3 \%)$ & 9944 (40.9\%) & $12,442(40.1 \%)$ & $6573(39.8 \%)$ & \\
\hline Ethnicity & & & & & & $<0.001$ \\
\hline White & 18,268 (97.5\%) & $16,960(94.7 \%)$ & I5,777 (96.2\%) & 20,517 (95.3\%) & 9737 (88.3\%) & \\
\hline Asian & 304 (I.6\%) & 710 (4.0\%) & 449 (2.7\%) & 769 (3.6\%) & 933 (8.5\%) & \\
\hline Black & $45(0.2 \%)$ & $104(0.6 \%)$ & $61(0.4 \%)$ & $143(0.7 \%)$ & $185(1.7 \%)$ & \\
\hline Mixed & $48(0.3 \%)$ & $78(0.4 \%)$ & $59(0.4 \%)$ & $57(0.3 \%)$ & $55(0.5 \%)$ & \\
\hline Other & 79 (0.4\%) & $66(0.4 \%)$ & $50(0.3 \%)$ & $47(0.2 \%)$ & 115 (1.0\%) & \\
\hline BMI $\left(\mathbf{k g} / \mathrm{m}^{2}\right)$ & $27.8(5.8)$ & $27.9(5.9)$ & $28.2(6.1)$ & $28.6(6.1)$ & $28.9(6.4)$ & $<0.001$ \\
\hline $\begin{array}{l}\text { Alcohol Consumption (Weekly } \\
\text { Units) }\end{array}$ & $4.0(0.0,10.0)$ & $3.0(0.0,10.0)$ & $2.0(0.0,10.0)$ & $2.0(0.0,10.0)$ & $2.0(0.0,10.0)$ & $<0.001$ \\
\hline Smoking Status & & & & & & $<0.001$ \\
\hline Never-Smoker & $16,376(59.2 \%)$ & I4,977 (57.3\%) & I2,492 (55.0\%) & $16,135(53.1 \%)$ & 7929 (50.8\%) & \\
\hline Ex-Smoker & 7672 (27.7\%) & $7483(28.6 \%)$ & $6549(28.9 \%)$ & 8744 (28.8\%) & 4206 (26.9\%) & \\
\hline Current Smoker & $3633(13.1 \%)$ & 3690 (14.1\%) & $3657(16.1 \%)$ & $5511(18.1 \%)$ & 3473 (22.3\%) & \\
\hline \multicolumn{7}{|l|}{ Comorbidities } \\
\hline Allergic Rhinitis & 2311 (8.2\%) & 2534 (9.4\%) & $2346(9.6 \%)$ & 3495 (11.3\%) & 2057 (12.4\%) & $<0.001$ \\
\hline Cancer & $3420(12.1 \%)$ & 2861 (10.6\%) & $2360(9.7 \%)$ & 3104 (10.0\%) & $1376(8.3 \%)$ & $<0.001$ \\
\hline Cataract & $347(1.2 \%)$ & $358(1.3 \%)$ & $374(1.5 \%)$ & $44 \mathrm{I}(\mathrm{I} .4 \%)$ & $259(1.6 \%)$ & 0.009 \\
\hline Cerebrovascular Disease & $427(1.5 \%)$ & $390(1.4 \%)$ & $369(1.5 \%)$ & $480(1.5 \%)$ & $231(1.4 \%)$ & 0.713 \\
\hline Congestive Heart Disease & $158(0.6 \%)$ & $132(0.5 \%)$ & $157(0.6 \%)$ & $197(0.6 \%)$ & $106(0.6 \%)$ & 0.092 \\
\hline Depression/Anxiety & 2615 (9.3\%) & 2440 (9.1\%) & $2181(9.0 \%)$ & 3441 (11.1\%) & 2021 (12.2\%) & $<0.001$ \\
\hline Diabetes & 1615 (5.7\%) & 1609 (6.0\%) & 1587 (6.5\%) & $2318(7.5 \%)$ & I396 (8.4\%) & $<0.001$ \\
\hline Eczema & 3286 (11.6\%) & 2789 (10.4\%) & 2447 (10.1\%) & $3310(10.7 \%)$ & $1718(10.4 \%)$ & $<0.001$ \\
\hline Glaucoma & $264(0.9 \%)$ & $250(0.9 \%)$ & $269(1.1 \%)$ & $381(1.2 \%)$ & $195(1.2 \%)$ & $<0.001$ \\
\hline Hypertension & 3362 (11.9\%) & $288 \mathrm{I}(10.7 \%)$ & $2342(9.6 \%)$ & 3904 (I2.6\%) & 2065 (12.5\%) & $<0.001$ \\
\hline Insomnia & $599(2.1 \%)$ & $659(2.4 \%)$ & $533(2.2 \%)$ & $669(2.2 \%)$ & $421(2.5 \%)$ & 0.005 \\
\hline Liver Disease & $40(0.1 \%)$ & $32(0.1 \%)$ & $47(0.2 \%)$ & $51(0.2 \%)$ & $28(0.2 \%)$ & 0.265 \\
\hline Myocardial Infarction & $167(0.6 \%)$ & $106(0.4 \%)$ & $123(0.5 \%)$ & $155(0.5 \%)$ & $86(0.5 \%)$ & 0.027 \\
\hline Nasal Polyps & $304(1.1 \%)$ & $239(0.9 \%)$ & $218(0.9 \%)$ & $279(0.9 \%)$ & $134(0.8 \%)$ & 0.034 \\
\hline Oral Candidiasis & $443(1.6 \%)$ & $436(1.6 \%)$ & $387(1.6 \%)$ & $630(2.0 \%)$ & $325(2.0 \%)$ & $<0.001$ \\
\hline Osteoporosis & $367(1.3 \%)$ & $324(1.2 \%)$ & 271 (1.1\%) & $339(1.1 \%)$ & $195(1.2 \%)$ & 0.154 \\
\hline Renal Disease & $856(3.0 \%)$ & $837(3.1 \%)$ & $741(3.0 \%)$ & 1155 (3.7\%) & $628(3.8 \%)$ & $<0.001$ \\
\hline Rheumatological Disease & $430(1.5 \%)$ & $368(1.4 \%)$ & $341(1.4 \%)$ & $425(1.4 \%)$ & $217(1.3 \%)$ & 0.345 \\
\hline Atopic Disease & 4258 (15.1\%) & 4105 (15.3\%) & $3938(16.2 \%)$ & 4919 (15.8\%) & $2673(16.2 \%)$ & $<0.001$ \\
\hline ICS Dose (BDP Equivalent) & $500(400,1000)$ & $500(400,1000)$ & $500(400,1000)$ & $500(400,1000)$ & $500(400,1000)$ & $<0.001$ \\
\hline LTRA & | 480 (5.2\%) & 1517 (5.6\%) & 1320 (5.4\%) & $1633(5.3 \%)$ & $922(5.6 \%)$ & 0.160 \\
\hline SABA & $23,707(84.0 \%)$ & $22,673(84.3 \%)$ & $20,093(82.6 \%)$ & 26,198 (84.3\%) & 13,777 (83.3\%) & $<0.001$ \\
\hline
\end{tabular}

(Continued) 
Table I (Continued).

\begin{tabular}{|c|c|c|c|c|c|c|}
\hline & $\begin{array}{c}5 \text { (Least } \\
\text { Deprived) }\end{array}$ & 4 & 3 & 2 & $\begin{array}{c}\text { I (Most } \\
\text { Deprived) }\end{array}$ & $\begin{array}{c}\text { P- } \\
\text { value }\end{array}$ \\
\hline Treatment Step (GINA 20I8) & & & & & & $<0.001$ \\
\hline 1 & $4699(16.7 \%)$ & $4579(17.0 \%)$ & $3784(15.6 \%)$ & $4972(16.0 \%)$ & $2806(17.0 \%)$ & \\
\hline 2 & $8847(31.4 \%)$ & $8425(31.3 \%)$ & $7782(32.0 \%)$ & $9592(30.9 \%)$ & $5273(31.9 \%)$ & \\
\hline 3 & $6425(22.8 \%)$ & $6069(22.6 \%)$ & $5653(23.2 \%)$ & $6862(22.1 \%)$ & $3759(22.7 \%)$ & \\
\hline 4 & 7828 (27.7\%) & $7373(27.4 \%)$ & $6779(27.9 \%)$ & 9191 (29.6\%) & $4446(26.9 \%)$ & \\
\hline 5 & $416(1.5 \%)$ & $454(1.7 \%)$ & $334(1.4 \%)$ & $442(1.4 \%)$ & $250(1.5 \%)$ & \\
\hline Blood Eosinophils ( $\left.10^{9} / \mathrm{L}\right)$ & $0.20(0.11,0.31)$ & $0.20(0.11,0.30)$ & $0.20(0.10,0.30)$ & $\begin{array}{c}0.20 \\
(0.1 I, 0.3 I)\end{array}$ & $0.20(0.11,0.31)$ & 0.001 \\
\hline Peak Flow (\%) & $89.0(76.1,100.8)$ & $\begin{array}{c}88.9 \\
(75.8,100.8)\end{array}$ & $\begin{array}{c}88.2 \\
(74.6,100.2)\end{array}$ & $\begin{array}{c}86.9 \\
(73.6,99.4)\end{array}$ & $84.9(70.9,97.3)$ & $<0.001$ \\
\hline Treatment Adherent (MPR $\geq 70 \%$ ) & $5920(25.6 \%)$ & $5674(26.2 \%)$ & $5185(26.4 \%)$ & $6886(27.5 \%)$ & $4173(31.6 \%)$ & $<0.001$ \\
\hline Asthma Review & 13,479 (47.8\%) & I I,852 (44.1\%) & $9764(40.1 \%)$ & $12842(4 \mid .3 \%)$ & 7114 (43.0\%) & $<0.001$ \\
\hline Uncontrolled Disease & $3228(34.5 \%)$ & $3034(35.8 \%)$ & 2761 (38.2\%) & $4130(40.1 \%)$ & $2121(44.7 \%)$ & $<0.001$ \\
\hline Any Exacerbations & $5732(20.3 \%)$ & $5734(21.3 \%)$ & $5024(20.6 \%)$ & 7261 (23.4\%) & 3996 (24.2\%) & $<0.001$ \\
\hline Respiratory Referral & $1226(4.3 \%)$ & $619(2.3 \%)$ & 767 (3.2\%) & $1094(3.5 \%)$ & $665(4.0 \%)$ & $<0.001$ \\
\hline
\end{tabular}

pronounced among those aged $>75$ years $(\mathrm{OR}=1.81,95 \%$ CI: $1.20,2.73)$ compared to those aged $<35$ years (OR: 1.22, 95\% CI: 0.85, 1.74; $\mathrm{p}=0.019)$. We also observed a stronger effect of SES on patients from ethnic minority groups (Figure 3). For EMGs, those in the most deprived quintile were more likely to have an exacerbation during follow-up (OR: 1.94, 95\% CI: 1.40, 2.68) when compared to those in the least deprived quintile. This was greater than the disparity for White patients (OR: 1.24, 95\% CI: $1.10,1.39 ; \mathrm{p}=0.012)$. There was a trend for a greater effect of deprivation on asthma disease control among EMGs (OR: 1.74, 95\% CI: 1.16, 2.63) when compared to White patients (OR: 1.35, 95\% CI: 1.00, 1.82), although this did not reach statistical significance $(p=0.294)$. We also found a greater effect of deprivation on peak flow among EMGs (ratio: 0.91, 95\% CI: 0.87, 0.96) when compared to White patients (ratio: 0.96 , 95\% CI: 0.94 , $0.98 ; \mathrm{p}=0.030)$.

When restricted to EMG patients, a comparison of IMD quintiles did not reveal any large differences in demographic presentation (Online Supplement 5). A larger proportion of patients in the most deprived quintile were Black or Asian $(86.8 \%$ vs $73.4 \%, p<0.001)$, and patients were less likely to be treated at GINA treatment step 3 or 4 (44.4\% vs $52.5 \%, \mathrm{p}<0.001)$, compared to those in the least deprived quintile. We also observed substantial differences in percent predicted peak flow measurements (78.5\% vs $85.5 \%, \mathrm{p}<0.001)$, proportion experiencing an exacerbation $(25.9 \%$ vs $16.8 \%, \mathrm{p}<0.001)$, and proportion uncontrolled $(54.8 \%$ vs $40.1 \%, \mathrm{p}<0.001)$ when comparing the most and least deprived quintiles. We found little evidence of any moderating influence of sex or asthma treatment step on the effect of SES (data not shown).

\section{Sensitivity Analyses}

Our conclusions were robust when using multiple imputation with chained equations to account for missing data, and when additionally adjusting for lifestyle factors (BMI and smoking status) in the multivariable regression models (Online Supplement 6). Respiratory referrals were more common among patients with at least two exacerbations in the previous year $(7.3 \%$ vs $3.4 \%$ in the entire cohort); however, we did not detect any difference by socioeconomic status with similar proportion referred in the lowest and highest IMD quintile $(8.0 \%$ vs $7.6 \% ; \mathrm{p}=0.915)$.

\section{Discussion}

In a large population-based cohort comprising 127,040 patients with asthma from UK primary care, we identified that more deprived patients had worse disease control, lower peak flow, and increased odds of an asthma exacerbation; however, there was no evidence of poorer 


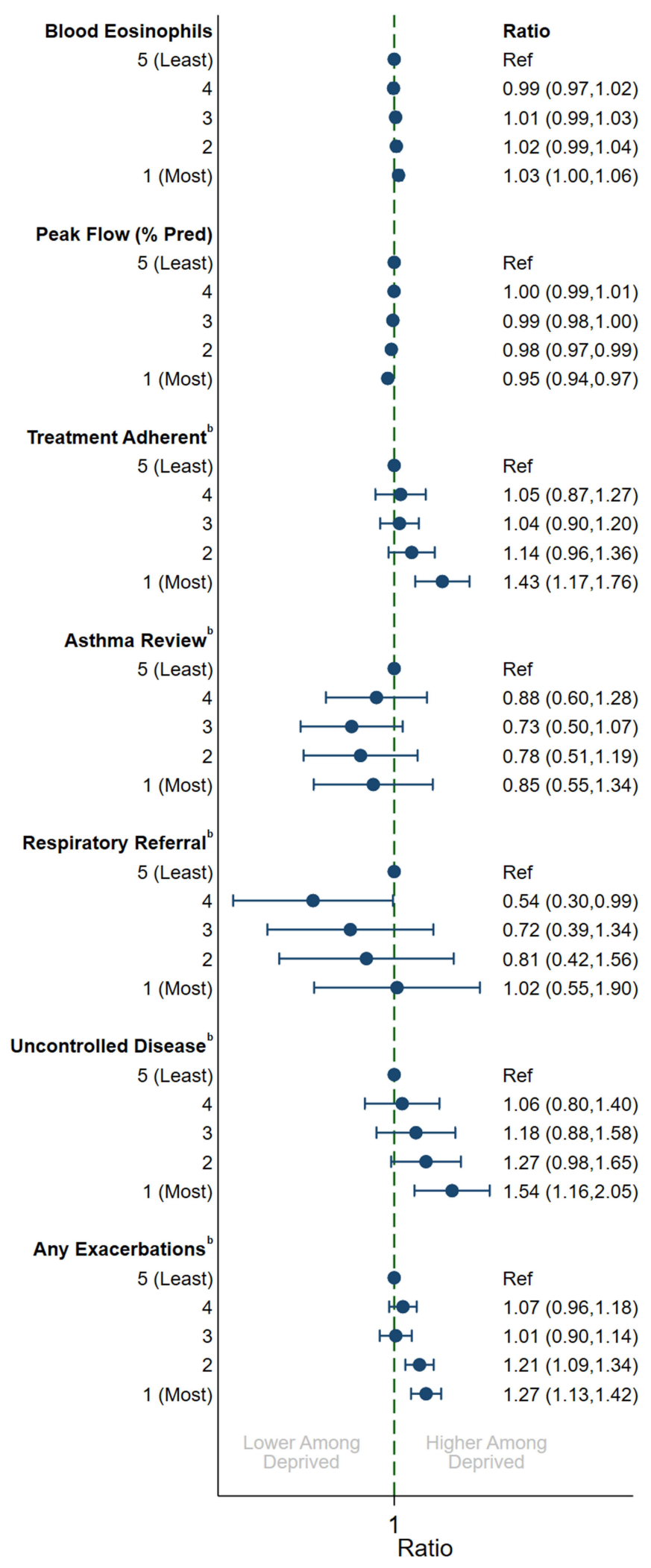

Figure I Multivariable association between indices of multiple deprivation quintile and clinical variables ${ }^{\text {a. a }}$ Adjusted for year, age (5-year groups) and sex, ${ }^{b}$ Odds ratio. medication adherence. There was evidence that the impact of deprivation was modulated in specific patient subgroups. In particular, socioeconomic disparities were greater among older patients (aged $>75$ years) and those from EMGs (Asian, Black, mixed, or other ethnicity). We did not find any evidence that these disparities were related to differences in medication adherence or in lifestyle factors such as BMI and smoking status.

Our findings of increased morbidity among more deprived patients with asthma is concerning and reflects potential disparities within the UK health-care system. Exploring the interaction between asthma health-care outcomes and socioeconomic status has been identified as a key research priority, and reducing disparities is a key aim of several health-care systems worldwide. ${ }^{36}$ Within the UK, health-care commissioners have a legal duty to consider the need to reduce inequalities. ${ }^{37}$ However, despite this obligation, our findings of poorer outcomes among deprived patients are consistent with a large body of previous research in asthma. Previous studies have consistently reported higher secondary health-care utilization (including emergency department [ED] attendances and hospitalizations) and morbidity (including exacerbations and mortality) among more deprived populations. ${ }^{10-12}$ Although much of this evidence is drawn from the US, these findings were consistent in countries with universally funded health-care systems such as the UK, Canada, and Australia. ${ }^{38}$

We have investigated the mediating effect of lifestyle factors such as smoking and BMI but did not find these to be a substantial driver of worse outcomes. Poorer medication adherence is frequently cited as a potential contributor to poorer asthma outcomes among disadvantaged groups, although evidence on the link between adherence and SES is inconsistent. ${ }^{39}$ We found better adherence among the most deprived quintile of patients; however, it should be noted that we used the MPR to estimate adherence, which accurately captures ICS prescriptions, but may not correspond to the collection and effective use of these medications by patients. ${ }^{40}$ Medications are inexpensive within the UK; they are free of charge to those receiving state benefits (including pensions) and require only a small $(<£ 10[\$ 14])$ co-payment for other patients, so substantial financial barriers to medication adherence are unlikely to exist among more deprived populations. In that context, it may be the case that higher medication use reflects the poorer symptom control observed within the lower 


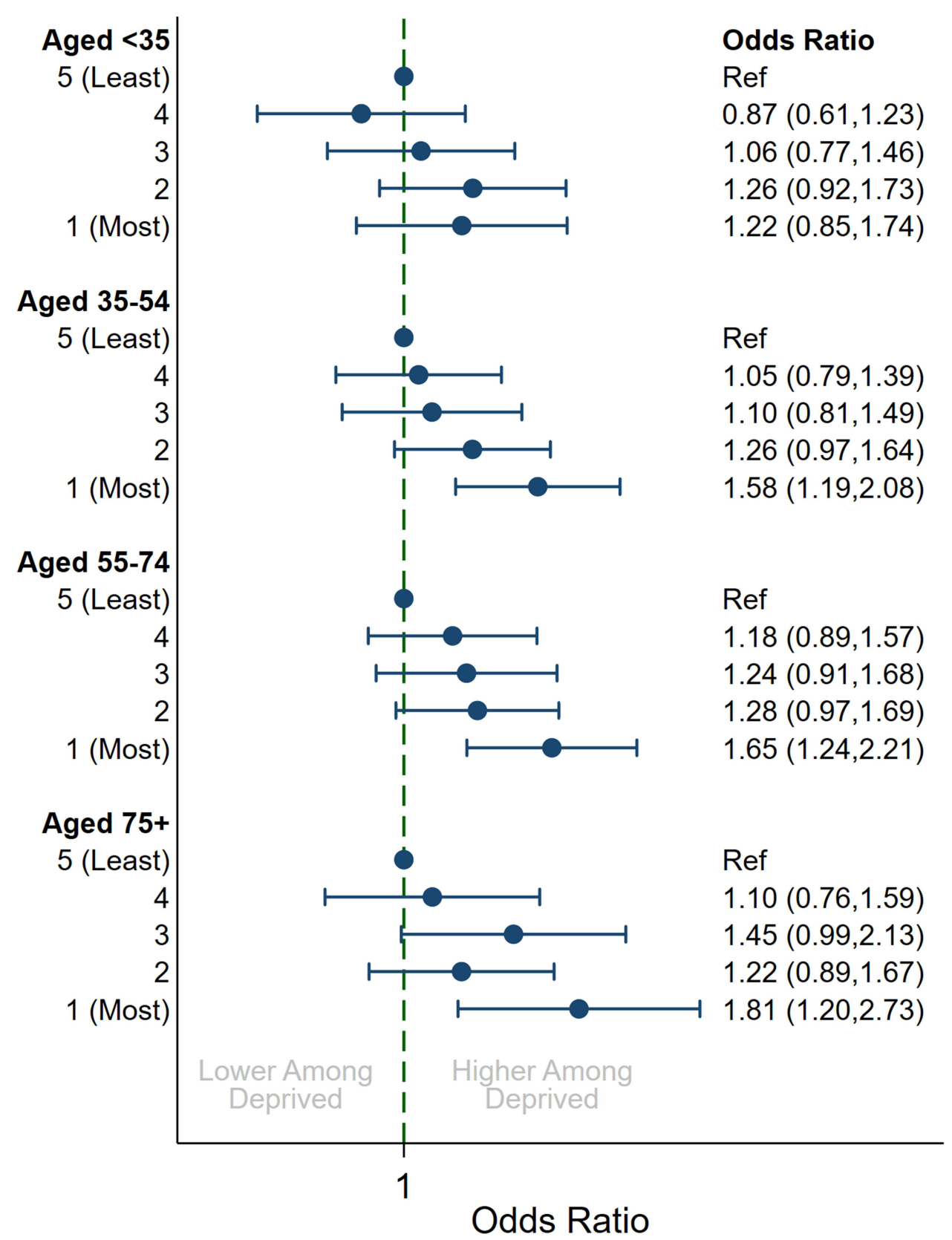

Figure 2 Multivariable association between indices of multiple deprivation quintile and uncontrolled disease by age group ${ }^{\mathrm{a} .}{ }^{\mathrm{a}}$ Adjusted for year, age (5-year groups) and sex.

SES patients in our study as reported elsewhere. ${ }^{41}$ We were unable to explore the impact of several potentially important mechanisms in our study, including the mediating role of poorer living conditions, increased propensity to access unscheduled care, and poorer health literacy, which has previously been reported among lower SES groups. ${ }^{42-44}$

Resolving socioeconomic disparities is unlikely to be straightforward. Interventions explored to improve health literacy have demonstrated promise as a means to reduce disparities, however, further consideration of the costs of providing these interventions and an exploration of their impact on health-care outcomes is required. ${ }^{45}$ Other issues, such as poorer housing conditions, are likely to require far-reaching and costly public interventions to resolve. Recent evidence from the US suggests that the childhood SES status of a parent had an effect on their children's asthma outcomes independent of their current economic circumstances, ${ }^{46}$ suggesting that the time scales required to fully address inequalities are likely to be generational. 
A

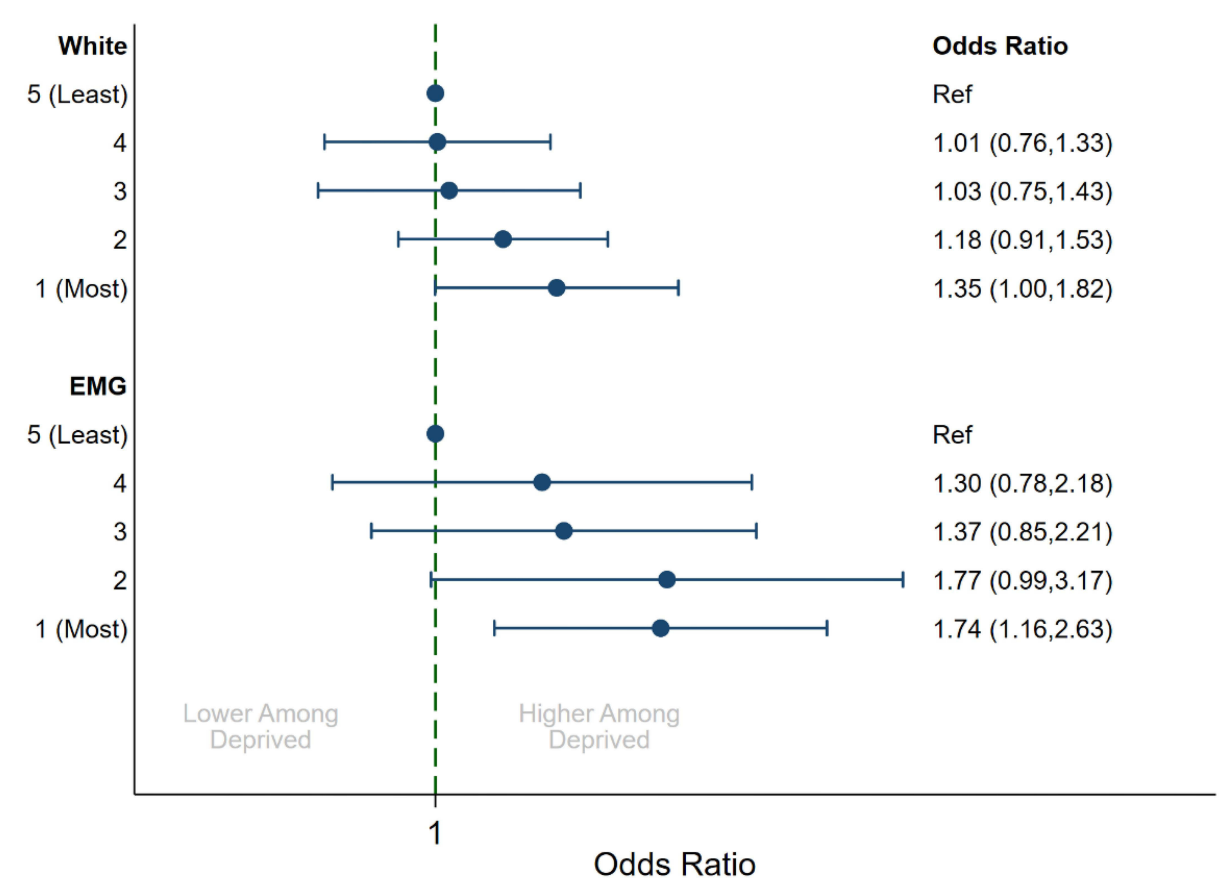

B

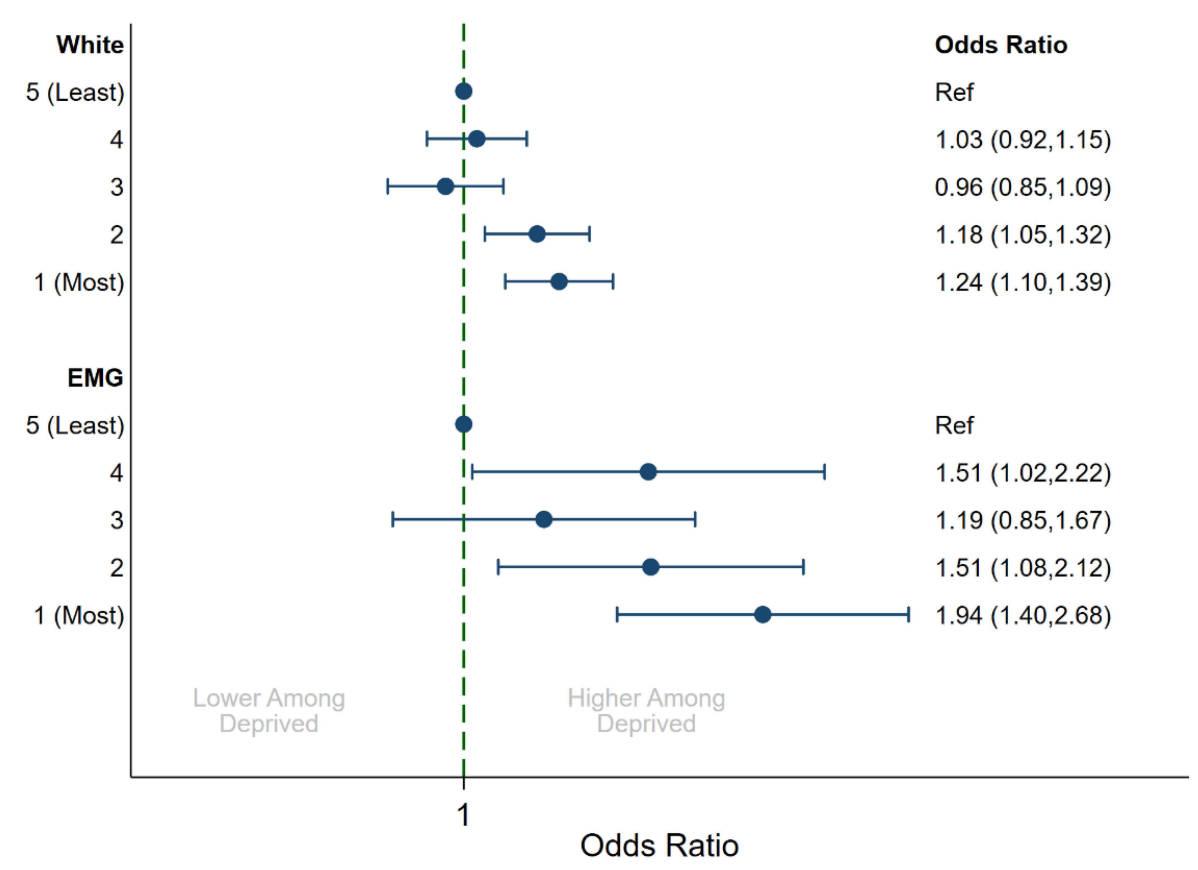

Figure 3 Multivariable association between indices of multiple deprivation quintile with uncontrolled disease and exacerbations by ethnicity ${ }^{\mathrm{a}}$. (A) Uncontrolled disease (B)

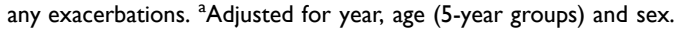

Our finding of increased socioeconomic disparities among specific patient subgroups suggests that targeted interventions are required to reduce these disparities. Prior studies have demonstrated markedly poorer outcomes among patients from EMGs, ${ }^{47-49}$ and this study provides evidence that considerable socioeconomic disparities also exist within this subgroup. We were unable to fully explore the reasons for this; however, it may be 
that issues relating to health literacy or poorer living conditions (including increased allergen exposure, air pollution, and overcrowding) are exacerbated among deprived EMG patients who are more likely to face language barriers or structural inequality in housing provision. ${ }^{50,51}$ It is notable that the most deprived patients from EMGs received lower doses of ICS despite substantially poorer disease control, which may reflect under-treatment in this patient group. Medication adherence is frequently lower among EMG patients; ${ }^{50,51}$ however, we found slightly higher adherence among the most deprived EMG patients when compared to the least deprived EMG patients, suggesting this did not play in an important role in driving poorer outcomes. The interaction between ethnicity and deprivation is an important area for future research, and it is likely to require tailored and culturally appropriate interventions to reduce inequalities. ${ }^{52,53}$

Our finding of a larger disparity among older aged patients is in concordance with a recent UK-based study of asthma mortality, ${ }^{18}$ and could be related to long-term effects of poorer disease control, smoking or occupational exposures during their lifetime.

The main strength of our study lies in its use of realworld population-based data that is reflective of asthma treatment in the UK. The large size of the dataset enabled the robust investigation of the socioeconomic differences within specific patient subgroups such as EMGs. Our study does have several potential limitations. It is observational and hence open to confounding due to unmeasured or poorly measured factors. Data were frequently missing for some of the variables included in our analysis; however, we believe it is unlikely that this would act differentially by socioeconomic status and, reassuringly, our conclusions were unchanged when using multiple imputation with chained equations to account for missing data. The Indices of Multiple Deprivation is an area-based measure derived from the postcode of the patient's general practice, which may not accurately reflect the circumstances of the individual patients included in our analysis. ${ }^{54,55}$ However, this potential misallocation should manifest in an underestimate of SES disparities due to regression dilution bias, meaning our main conclusions are not affected. Finally, there were a relatively low number of patients from EMGs, which did not facilitate a comparison of whether SES disparities differed by specific ethnicity (eg, Asian, Black). It is likely that differences will exist between ethnic minority subgroups within the UK as shown elsewhere. ${ }^{48}$

\section{Conclusions}

Patients from more deprived areas had poorer asthma disease control, lower peak flow, and increased exacerbations. There was evidence that the magnitude of socioeconomic disparities were elevated among older patients and those from ethnic minority groups. We did not find any evidence that poorer medication adherence or differences in smoking rates played an important role in driving disparities; however, the drivers of these differences require further exploration.

\section{Abbreviations}

BMI, body mass index; ED, emergency department; EMG, ethnic minority group; GINA, Global Initiative for Asthma; ICS, inhaled corticosteroids; IMD, indices of multiple deprivation; LABA, long-acting $\beta$-agonist; LAMA, long-acting muscarinic antagonist; LTRA, leukotriene receptor antagonists; OCS, oral corticosteroids; OPCRD, Optimum Patient Care Research Database; OR, odds-ratio; SABA, short-acting $\beta$-agonist; SES, socioeconomic status.

\section{Data Sharing Statement}

The dataset supporting the conclusions of this article was derived from the Optimum Patient Care Research Database (www.opcrd.co.uk). The OPCRD has ethical approval from the National Health Service (NHS) Research Authority to hold and process anonymised research data (Research Ethics Committee reference: 15/ EM/0150). This study was approved by the Anonymised Data Ethics Protocols and Transparency (ADEPT) committee (ADEPT approval reference: ADEPT0120) - the independent scientific advisory committee for the OPCRD. The authors do not have permission to give public access to the study dataset; researchers may request access to OPCRD data for their own purposes. Access to OCPRD can be made via the OCPRD website (https:// opcrd.co.uk/our-database/data-requests/) or via the enquiries email info@opcrd.co.uk.

\section{Ethical Approval}

OPCRD has been reviewed and ethically approved by the NHS Health Research Authority to hold and process anonymized data as part of service delivery (Research Ethics Committee reference: 15/EM/0150). Specific approval for this research study was granted by the Anonymised Data Ethics Protocols and Transparency committee (ADEPT 
approval reference: ADEPT0120). This study was designed, implemented, and registered in accordance with the criteria of the European Network of Centres for Pharmacoepidemiology and Pharmacovigilance (EUPAS32482).

\section{Acknowledgments}

We would like to acknowledge Ms. Audrey Ang (BSc, Hons) of the Observational and Pragmatic Research Institute (OPRI), Singapore, for editorial and formatting assistance that supported the development of this publication.

\section{Funding}

This research study was funded and delivered by the Observational \& Pragmatic Research Institute Pte Ltd (OPRI). Optimum Patient Care Global received partial funding from AstraZeneca Ltd to support dataset creation.

\section{Disclosure}

John Busby declares no relevant conflicts of interest. David Price has board membership with AstraZeneca, Boehringer Ingelheim, Chiesi, Mylan, Novartis, Regeneron Pharmaceuticals, Sanofi Genzyme, Thermofisher; consultancy agreements with Airway Vista Secretariat, AstraZeneca, Boehringer Ingelheim, Chiesi, EPG Communication Holdings Ltd, FIECON Ltd, Fieldwork International, GlaxoSmithKline, Mylan, Mundipharma, Novartis, OM Pharma SA, PeerVoice, Phadia AB, Spirosure Inc, Strategic North Limited, Synapse Research Management Partners S.L., Talos Health Solutions, Theravance and WebMD Global LLC; grants and unrestricted funding for investigator-initiated studies (conducted through Observational and Pragmatic Research Institute Pte Ltd) from AstraZeneca, Boehringer Ingelheim, Chiesi, Mylan, Novartis, Regeneron Pharmaceuticals, Respiratory Effectiveness Group, Sanofi Genzyme, Theravance and UK National Health Service; payment for lectures/speaking engagements from AstraZeneca, Boehringer Ingelheim, Chiesi, Cipla, GlaxoSmithKline, Kyorin, Mylan, Mundipharma, Novartis, Regeneron Pharmaceuticals and Sanofi Genzyme; payment for travel/accommodation/meeting expenses from AstraZeneca, Boehringer Ingelheim, Mundipharma, Mylan, Novartis, Thermofisher; stock/ stock options from AKL Research and Development Ltd which produces phytopharmaceuticals; owns 74\% of the social enterprise Optimum Patient Care Ltd (Australia and UK) and 92.61\% of Observational and Pragmatic Research Institute Pte Ltd (Singapore); 5\% shareholding in Timestamp which develops adherence monitoring technology; is peer reviewer for grant committees of the UK Efficacy and Mechanism Evaluation programme, and Health Technology Assessment; and was an expert witness for GlaxoSmithKline. Riyad Al-Lehebi has given lectures at meetings supported by AstraZeneca, Boehringer Ingelheim, GlaxoSmithKline, Novartis, and Sanofi and participated in advisory board fees from GlaxoSmithKline. Sinthia Bosnic-Anticevich has received honorarium for participation in expert advisory boards and given lectures for Teva Pharmaceuticals, AstraZeneca, Boehringer Ingelheim, GSK, Meda, Mundipharma, Sanofi, Mylan and received unrestricted research grants from Mylan, AstraZeneca, Teva and Mundipharma International. Job F.M. van Boven and/or his institution received grants and consultancy fees from AstraZeneca, Chiesi, GSK, Boehringer Ingelheim, Pill Connect, TEVA, Novartis, Trudell Medical, Menarini, European Commission (COST Action 19132), and Aardex. Benjamin Emmanuel is an employee of AstraZeneca, a co-funder of the International Severe Asthma Registry. J. Mark FitzGerald reports grants from AstraZeneca, GSK, Sanofi Regeneron, Novartis paid directly to UBC. Personal fees for lectures and attending advisory boards: Astra Zeneca, GSK, Sanofi Regeneron, Novartis, TEVA. Mina Gaga reports grants and personal fees from Novartis, BMS, and Menarini; grants from Galapagos and Elpen; and personal fees from MSD and Roche outside the submitted work. Susanne Hansen reports no conflict of interest. Mark Hew declares grants and other advisory board fees (made to his institutional employer) from AstraZeneca, GlaxoSmithKline, Novartis, Sanofi, and Seqirus, Teva, for unrelated projects. Takashi Iwanaga declares grants from Astellas, Boehringer Ingelheim, Daiichi-Sankyo, Kyorin, MeijiSeika Pharma, Novartis, Teijin Pharma, Ono, Otsuka Pharmaceutical Factory, and Taiho, and lecture fees from Kyorin, GlaxoSmithKline, and AstraZeneca.

Désirée Larenas Linnemann reports personal fees from Amstrong, AstraZeneca, Boehringer Ingelheim, Carnot., Chiesi, Circassia, DBV Technologies, Grunenthal, GSK, MEDA, Menarini, MSD, Novartis, Pfizer, Novartis, Sanofi, Siegfried, UCB, Viatris, Alakos, Gossamer, grants 
from Sanofi, AstraZeneca, Novartis, UCB, GSK, TEVA, Boehringer Ingelheim, Chiesi, and Purina institute, outside the submitted work. Bassam Mahboub reports no conflict of interest. Patrick Mitchell has received speaker fees from GlaxoSmithKline, AstraZeneca, Teva, and Novartis, and has received grants from AstraZeneca and Teva. Daniela Morrone reports no relevant conflicts of interest. Jonathan Pham reports no relevant conflicts of interest. Celeste Porsbjerg has attended advisory boards for AstraZeneca, Novartis, TEVA, and Sanofi-Genzyme; has given lectures at meetings supported by AstraZeneca, Novartis, TEVA, Sanofi-Genzyme, and GlaxoSmithKline; has taken part in clinical trials sponsored by AstraZeneca, Novartis, MSD, Sanofi-Genzyme, GlaxoSmithKline, and Novartis; and has received educational and research grants from AstraZeneca, Chiesi, Novartis, TEVA, GlaxoSmithKline, ALK, and Sanofi-Genzyme. Nicolas Roche reports grants and personal fees from Boehringer Ingelheim, Novartis, and Pfizer, and personal fees from Teva, GSK, AstraZeneca, Chiesi, Sanofi, and Zambon. Eileen Wang has received honoraria from AstraZeneca, Clinical Care Options, Optimum Patient Care, and Sema4. She has been an investigator on clinical trials sponsored by AstraZeneca, GlaxoSmithKline, Genentech, Novartis, Teva, and National Institute of Allergy and Infectious Diseases (NIAID) for which her institution has received funding. Neva Eleangovan is an employee of the Observational and Pragmatic Research Institute, which conducted this study in collaboration with Optimum Patient Care and AstraZeneca. Liam G. Heaney declares he has received grant funding, participated in advisory boards and given lectures at meetings supported by Aerocrine and Vitalograph, Amgen, AstraZeneca, Boehringer Ingelheim, Chiesi, Circassia, Evelo Biosciences, Hoffmann la Roche, GlaxoSmithKline, MedImmune, Napp Pharmaceuticals, Novartis, Sanofi, Theravance and Teva; he has taken part in asthma clinical trials sponsored by Boehringer Ingelheim, Hoffmann la Roche, and GlaxoSmithKline for which his institution received remuneration; he is the Academic Lead for the Medical Research Council Stratified Medicine UK Consortium in Severe Asthma which involves industrial partnerships with a number of pharmaceutical companies including Amgen, AstraZeneca, Boehringer Ingelheim, GlaxoSmithKline, Hoffmann la Roche, and Janssen. The authors report no other conflicts of interest in this work.

\section{References}

1. Office for National Statistics. Health state life expectancies by national deprivation deciles, England: 2017 to 2019, 2021.

2. Soriano JB, Abajobir AA, Abate KH, et al. Global, regional, and national deaths, prevalence, disability-adjusted life years, and years lived with disability for chronic obstructive pulmonary disease and asthma, 1990-2015: a systematic analysis for the Global Burden of Disease Study 2015. Lancet Respir Med. 2017;5(9):691-706. doi:10.1016/S2213-2600(17)30293-X

3. Nurmagambetov T, Kuwahara R, Garbe P. The economic burden of asthma in the United States, 2008-2013. Ann Am Thorac Soc. 2018;15(3):348-356. doi:10.1513/AnnalsATS.201703-259OC

4. Uphoff E, Cabieses B, Pinart M, et al. A systematic review of socioeconomic position in relation to asthma and allergic diseases. Eur RespirJ. 2015;46(2):364-374. doi:10.1183/09031936.00114514

5. Asthma UK. On the Edge: How Inequality Affects People with asthma. London; 2018.

6. Masefield S, Edwards J, Hansen K, et al. The future of asthma research and development: a roadmap from the European Asthma Research and Innovation Partnership (EARIP). Eur Respir J. 2017;49 (5):1602295. doi:10.1183/13993003.02295-2016

7. Sullivan K, Thakur N. Structural and social determinants of health in asthma in developed economies: a scoping review of literature published between 2014 and 2019. Curr Allergy Asthma Rep. 2020;20:2. doi:10.1007/s11882-020-0899-6

8. Laba TL, Jan S, Zwar NA, et al. Cost-related underuse of medicines for asthma-opportunities for improving adherence. J Allergy Clin Immunol-Pract. 2019;7(7):2298-+. doi:10.1016/j.jaip.2019.03.024

9. Dickman SL, Himmelstein DU, Woolhandler S, et al. Inequality and the health-care system in the USA. Lancet. 2017;389(10077):14311441. doi:10.1016/S0140-6736(17)30398-7

10. Eisner MD, Katz PP, Yelin EH, et al. Risk factors for hospitalization among adults with asthma: the influence of sociodemographic factors and asthma severity. Respir Res. 2001;2(1):53-60. doi:10.1186/rr37

11. Kaur BP, Lahewala S, Arora S, et al. Asthma: hospitalization trends and predictors of in-hospital mortality and hospitalization costs in the USA (2001-2010). Int Arch Allergy Immunol. 2015;168(2):71-78. doi:10.1159/000441687

12. Lutfiyya MN, McCullough JE, Lipsky MS. A population-based study of health service deficits for US adults with asthma. J Asthma. 2011;48(9):931-944. doi:10.3109/02770903.2011.619023

13. Backman H, Hedman L, Stridsman C, et al. A population-based cohort of adults with asthma: mortality and participation in a longterm follow-up. Eur Clin Respir J. 2017;4(1):1334508. doi:10.1080/ 20018525.2017.1334508

14. To T, Simatovic J, Zhu J, et al. Asthma deaths in a large provincial health system. A 10-year population-based study. Ann Am Thorac Soc. 2014;11(8):1210-1217. doi:10.1513/AnnalsATS.201404-138OC

15. Higgins BG, Britton JR. Geographical and social class effects on asthma mortality in England and Wales. Respir Med. 1995;89 (5):341-346. doi:10.1016/0954-6111(95)90005-5

16. Jones AP, Bentham G. Health service accessibility and deaths from asthma in 401 local authority districts in England and Wales, 198892. Thorax. 1997;52(3):218-222. doi:10.1136/thx.52.3.218

17. Wainwright NW, Surtees PG, Wareham NJ, et al. Psychosocial factors and incident asthma hospital admissions in the EPIC-Norfolk cohort study. Allergy. 2007;62(5):554-560. doi:10.1111/j.13989995.2007.01316.x

18. Gupta RP, Mukherjee M, Sheikh A, et al. Persistent variations in national asthma mortality, hospital admissions and prevalence by socioeconomic status and region in England. Thorax. 2018;73 (8):706-712. doi:10.1136/thoraxjnl-2017-210714 
19. OPCRD. OPCRD: our Databases; 2020 [cited May 21, 2020]. Available from: https://opcrd.co.uk/our-database/. Accessed May 20 2020.

20. Oneil M, Payne C, Read J. Read-codes version-3 - a user led terminology. Methods Inf Med. 1995;34(1-2):187-192.

21. Nissen F, Morales DR, Mullerova H, et al. Validation of asthma recording in the Clinical Practice Research Datalink (CPRD). BMJ Open. 2017;7(8):e017474. doi:10.1136/bmjopen-2017-017474

22. Di Martino M, Kirchmayer U, Agabiti N, et al. The impact of timewindow bias on the assessment of the long-term effect of medication adherence: the case of secondary prevention after myocardial infarction. BMJ Open. 2015;5(6):e007866. doi:10.1136/bmjopen-2015007866

23. Noble M, Wright G, Smith G, et al. Measuring multiple deprivation at the small-area level. Environ Planni. 2006;38(1):169-185. doi: $10.1068 / \mathrm{a} 37168$

24. Knudson RJ, Lebowitz MD, Holberg CJ, et al. Changes in the normal maximal expiratory flow-volume curve with growth and aging. $\mathrm{Am}$ Rev Respir Dis. 1983;127(6):725-734. doi:10.1164/ arrd.1983.127.6.725

25. Ryan D, Heatley H, Heaney LG, et al. Potential severe asthma hidden in UK primary care. J Allergy Clin Immunol Pract. 2021;9(4):161223.e9. doi:10.1016/j.jaip.2020.11.053

26. Thomas M, Gruffydd-Jones K, Stonham C, et al. Assessing asthma control in routine clinical practice: use of the Royal College of Physicians '3 questions. Prim Care Respir J. 2009;18(2):83-88. doi:10.3132/pcrj.2008.00045

27. Khan NF, Perera R, Harper S, et al. Adaptation and validation of the Charlson Index for Read/OXMIS coded databases. BMC Fam Pract. 2010;11(1):1. doi:10.1186/1471-2296-11-1

28. Morgan C, Webb RT, Carr MJ, et al. Incidence, clinical management, and mortality risk following self harm among children and adolescents: cohort study in primary care. BMJ. 2017;359:j4351. doi:10.1136/bmj.j4351

29. Sweeney J, Patterson CC, Menzies-Gow A, et al. Comorbidity in severe asthma requiring systemic corticosteroid therapy: cross-sectional data from the Optimum Patient Care Research Database and the British Thoracic Difficult Asthma Registry. Thorax. 2016;71 (4):339-346. doi:10.1136/thoraxjnl-2015-207630

30. Global Initiative for Asthma. Global Strategy for Asthma Management and Prevention, Updated; 2018.

31. NICE. Inhaled Corticosteroid Doses for NICE's Asthma Guideline; 2018.

32. Bloom CI, Nissen F, Douglas IJ, et al. Exacerbation risk and characterisation of the UK's asthma population from infants to old age. Thorax. 2018;73(4):313-320. doi:10.1136/thoraxjnl-2017-210650

33. Schisterman EF, Cole SR, Platt RW. Overadjustment bias and unnecessary adjustment in epidemiologic studies. Epidemiology 2009;20 (4):488495. doi: 10.1097/EDE.0b013e3181a819a1

34. Taylor DR, Bateman ED, Boulet L-P, et al. A new perspective on concepts of asthma severity and control. Eur Respir J. 2008;32 (3):545-554. doi:10.1183/09031936.00155307

35. BTS/SIGN. British Guideline on the Management of Asthma: Quick Reference Guide; 2019.

36. Commonwealth Fund. What is being done to reduce disparities?; 2021. Available from: https:/www.commonwealthfund.org/interna tional-health-policy-center/system-features/what-being-done-reducedisparities. Accessed October 12, 2021.

37. UK Government. Health and Social Care Act 2012; 2012.

38. The Commonwealth Fund. International Profiles of Health Care Systems; 2020.
39. Mazumdar S, Ghosh S, Mukherjee S. Non-adherence to asthma medications: relation to socioeconomic status and asthma education. Eur Respir J. 2015;46. doi:10.1183/13993003.congress-2015. OA4792.

40. Lindsay JT, Heaney LG. Non-adherence in difficult asthma and advances in detection. Expert Rev Respir Med. 2013;7(6):607-614. doi:10.1586/17476348.2013.842129

41. van Boven JFM, Koponen M, Lalic S, et al. Trajectory analyses of adherence patterns in a real-life moderate to severe asthma population. J Allergy Clin Immunol Pract. 2020;8(6):1961-69.e6. doi:10.1016/j.jaip.2019.12.002

42. Willems S, Peersman W, De Maeyer P, et al. The impact of neighborhood deprivation on patients' unscheduled out-of-hours healthcare seeking behavior: a cross-sectional study. BMC Fam Pract. 2013;14:9. doi:10.1186/1471-2296-14-136

43. Stormacq C, Van den Broucke S, Wosinski J. Does health literacy mediate the relationship between socioeconomic status and health disparities? Integrative review. Health Promot Int. 2019;34(5):e1e17. doi:10.1093/heapro/day062

44. Hajat A, Hsia C, O’Neill MS. Socioeconomic disparities and air pollution exposure: a global review. Curr Environ Health Rep. 2015;2(4):440-450. doi:10.1007/s40572-015-0069-5

45. Walters R, Leslie SJ, Polson R, et al. Establishing the efficacy of interventions to improve health literacy and health behaviours: a systematic review. BMC Public Health 2020;20(1):1-7. doi: 10.1186/s12889-020-08991-0

46. Chen E, Shalowitz MU, Story RE, et al. Parents' childhood socioeconomic circumstances are associated with their children's asthma outcomes. J Allergy Clin Immunol 2017;140(3):828-35.e2. doi: 10.1016/j.jaci.2016.11.040

47. Netuveli G, Hurwitz B, Levy M, et al. Ethnic variations in UK asthma frequency, morbidity, and health-service use: a systematic review and meta-analysis. Lancet. 2005;365(9456):312-317.

48. Sheikh A, Steiner MFC, Cezard G, et al. Ethnic variations in asthma hospital admission, readmission and death: a retrospective, national cohort study of 4.62 million people in Scotland. BMC Med. 2016;14. doi:10.1186/s12916-015-0546-6

49. Hull SA, McKibben S, Homer K, et al. Asthma prescribing, ethnicity and risk of hospital admission: an analysis of 35,864 linked primary and secondary care records in East London. NPJ Prim Care Resp M. 2016;26. doi:10.1038/npjpcrm.2016.49

50. Race Equality Foundation. Ethnic Disadvantage in the Housing Market: Evidence from the 2011 Census; 2015.

51. The Migration Observatory. English Language Use and Proficiency of Migrants in the UK; 2019.

52. McQuaid EL. Barriers to medication adherence in asthma: the importance of culture and context. Ann Allergy Asthma Immunol. 2018;121 (1):37-42. doi:10.1016/j.anai.2018.03.024

53. Poureslami I, Nimmon L, Doyle-Waters M, et al. Effectiveness of educational interventions on asthma self-management in Punjabi and Chinese asthma patients: a randomized controlled trial. J Asthma. 2012;49(5):542-551. doi:10.3109/02770903.2012.682125

54. Pampalon R, Hamel D, Gamache P. A comparison of individual and area-based socio-economic data for monitoring social inequalities in health. Health Rep. 2009;20(4):85-94.

55. Ingleby FC, Belot A, Atherton I, et al. Assessment of the concordance between individual-level and area-level measures of socioeconomic deprivation in a cancer patient cohort in England and Wales. BMJ Open. 2020;10(11):e041714. doi:10.1136/bmjopen2020-041714 


\section{Publish your work in this journal}

The Journal of Asthma and Allergy is an international, peer-reviewed open-access journal publishing original research, reports, editorials and commentaries on the following topics: Asthma; Pulmonary physiology; Asthma related clinical health; Clinical immunology and the immunological basis of disease; Pharmacological interventions and new therapies. The manuscript management system is completely online and includes a very quick and fair peer-review system, which is all easy to use. Visit http://www.dovepress.com/testimonials.php to read real quotes from published authors.

Submit your manuscript here: https:/www.dovepress.com/journal-of-asthma-and-allergy-journal 